\title{
EchoGéo
}

ECHOGEO- $\quad 41 \mid 2017$

Un état des lieux du Brésil en 2017

\section{Le Brésil et ses Indiens : une réconciliation impossible?}

François-Michel Le Tourneau

\section{OpenEdition}

1 Journals

Electronic version

URL: https://journals.openedition.org/echogeo/15027

DOI: 10.4000/echogeo.15027

ISSN: 1963-1197

Publisher

Pôle de recherche pour l'organisation et la diffusion de l'information géographique (CNRS UMR 8586)

Electronic reference

François-Michel Le Tourneau, "Le Brésil et ses Indiens : une réconciliation impossible ?", EchoGéo [Online], 41 | 2017, Online since 28 September 2017, connection on 03 August 2021. URL: http:// journals.openedition.org/echogeo/15027 ; DOI: https://doi.org/10.4000/echogeo.15027

This text was automatically generated on 3 August 2021.

EchoGéo est mis à disposition selon les termes de la licence Creative Commons Attribution - Pas d'Utilisation Commerciale - Pas de Modification 4.0 International (CC BY-NC-ND) 


\title{
Le Brésil et ses Indiens : une réconciliation impossible?
}

\author{
François-Michel Le Tourneau
}

1 En ouvrant de larges droits fonciers aux Amérindiens, la Constitution de 1988 avait semblé avancer dans le sens de la réparation d'un préjudice historique, à savoir la dépossession des habitants originels du Brésil au profit d'abord des colons portugais puis de la société brésilienne. Malgré les débats lors de l'Assemblée constituante, la solution du " problème indien » semblait à portée de main avec l'affirmation dans la loi fondamentale du caractère pluriculturel et pluriethnique du Brésil (Boyer, 2010).

2 Trente ans plus tard, force est de constater que la réconciliation attendue n'a pas eu lieu, et que le contexte politique entourant les questions amérindiennes est presque totalement retourné. Il y a des raisons sociales à cela, magistralement analysées par Roberto da Matta (1976) ou Alcida Ramos (1998), en particulier l'écart immense entre l'Indien idéalisé qui est intégré au récit national brésilien et les Amérindiens réels, qui sont l'objet d'une discrimination qui demeure forte encore aujourd'hui. Mais le moteur principal de la dispute est sans aucun doute d'ordre spatial. Grâce à la définition extensive de leurs droits fonciers dans la nouvelle Constitution, les Amérindiens ont obtenu un usufruit exclusif sur une portion considérable du territoire brésilien (environ $13,7 \%^{1}$ ). Ils apparaissent de ce fait comme des concurrents pour certains secteurs politico-économiques, alors qu'en parallèle leur voix pèse de plus en plus dans les débats autour de la construction de grandes infrastructures, qu'ils peuvent désormais en théorie bloquer ou considérablement ralentir si elles ont un impact sur leurs terres.

3 Si les dispositions adoptées en 1988 pouvaient apparaître à l'origine comme des concessions spatiales destinées à maintenir un symbole un peu exotique de brésilianité, la question a donc évolué aujourd'hui et ce qui est en jeu est désormais la place concédée aux Amérindiens, population ultra-minoritaire sur le plan démographique, dans la définition de l'avenir du Brésil et de sa trajectoire de développement. Or le balancier semble avoir effectué son mouvement en sens inverse depuis une dizaine d'années, au point que la question d'un retour en arrière, dont les conséquences 
seraient profondes en termes de transformations géographiques, en particulier en Amazonie, est désormais envisageable.

4 Pour faire le point sur cette question et sur les enjeux qui lui sont liés, cet article propose tout d'abord un rapide panorama historique de la manière dont la question amérindienne a été considérée au Brésil depuis la colonisation portugaise jusqu'aux avancées de la fin du $\mathrm{XX}^{\mathrm{e}}$ siècle, en se focalisant principalement sur la question des territoires. Dans un deuxième temps, il montrera comment la Constitution de 1988 a permis la consolidation d'un patrimoine foncier très important, alors que la population amérindienne demeure ultra-minoritaire. La dernière partie fait le point sur la situation actuelle en essayant de montrer comment l'inflexion dans un sens défavorable aux droits fonciers des Amérindiens est patente depuis plus de dix ans et combien la situation actuelle met à l'épreuve les protections dont ils bénéficient. En conclusion, le texte s'interroge sur les scénarios possibles pour les prochaines années.

\section{Une présence tantôt niée, tantôt idéalisée}

5 Comme pour l'ensemble des Amériques, la catastrophe représentée par l'arrivée des Européens pour les Amérindiens du Brésil peut s'exprimer en quelques chiffres glaçants. Si Carlos Fausto (2000) estime entre 5 et 8 millions la population avant l'arrivée des Européens ${ }^{2}$, l'estimation du nombre des Amérindiens donnée par le Service de Protection des Indiens (SPI) au début des années 1950 était de 100000 personnes, faisant dire à Darcy Ribeiro (1970) que tous les Indiens du Brésil ne pourraient plus remplir (et de loin!) le stade du Maracanã... En écho à ce phénomène, sur le plan géographique, la rétraction des territoires amérindiens est continue jusqu'à la fin du XXe siècle. Cependant, à la différence d'autres pays comme l'Argentine ou le Chili, le Brésil fait une large part à sa composante amérindienne dans la définition de son identité nationale. Retracer rapidement l'histoire des relations entre le Brésil et ses Amérindiens permet de faire le point sur ce double mouvement assez paradoxal.

\section{Alliés, ennemis ou esclaves : le sort des Amérindiens dans le Brésil colonial}

6 Les premiers contacts des Portugais avec les Amérindiens du Brésil sont tantôt rugueux, tantôt amicaux. Dès les premières décennies de la colonie une cartographie se met en place qui distingue les Indiens amis (Índios mansos) des ennemis (Índios bravos), ce d'autant que les puissances européennes, qui n'ont que des ressources humaines limitées à investir en Amérique, poursuivent leurs luttes en enrôlant à leurs côtés telle ou telle ethnie en jouant des rivalités locales. Ainsi, lors de l'épisode de la France antarctique les Français compteront sur les Tupinambá alors que les Portugais feront appel aux Tupiniquim, et le changement d'alliance des Potiguar fut pour beaucoup dans la reprise du Pernambouco aux Hollandais au XVII siècle. De fait les Portugais se montrent particulièrement habiles dans ce jeu. Ils incluent souvent les Amérindiens au titre de supplétifs pour la protection des zones annexées, leur conférant grades, salaires, et éventuellement titres de noblesse (Pinto de Medeiros, 2011). Comme le souligne Beatriz Perone Moises (1992), le fait de bien traiter les groupes alliés et le respect de leur liberté est un des principes officiels constants de la colonisation portugaise (pas toujours respecté dans les faits, et de loin), qui se consolide 
juridiquement au fur et à mesure de l'histoire de la colonie, de même que le fait que les Indiens sont « seigneurs de leurs terres" (toute la question étant de savoir comment définir les limites de celles-ci).

Car le Brésil cependant fait face en parallèle à un cruel manque de main-d'œuvre pour produire les denrées demandées par la métropole (notamment les drogues du sertão ${ }^{3}$, le cacao ou le sucre). De ce fait, au respect de la liberté des Amérindiens alliés (bien que, on le verra plus loin, leur travail puisse toutefois être mobilisé) répond la possibilité de réduire en esclavage les Indiens hostiles en utilisant deux mécanismes, réglementés notamment par une ordonnance de 1680 : la pratique du resgate et celle de la "guerre juste ». Dans le premier cas, les esclaves étaient d'abord capturés par des Indiens d'une ethnie rivale puis «sauvés " par des marchands de main d'œuvre qui les rachetaient. Redevables de leur vie (puisque soustraits à un cannibalisme supposé), ces captifs pouvaient alors être vendus comme esclaves. Dans le second, en cas d'actions hostiles de la part d'un groupe amérindien, la couronne pouvait donner le droit de "pacifier " une région en effectuant des razzias sur les villages et en réduisant ces Indiens en captivité. La conjonction de ces deux mécanismes a permis une vaste chasse à l'homme au profit des planteurs et commerçants portugais. Ce fut notamment le cas pour la rive gauche de l'Amazone durant une grande partie du XVIII ${ }^{\mathrm{e}}$ siècle.

Un autre système de regroupement des Amérindiens est celui des missions, notamment jésuites, qui agrègent autour d'elles de nombreux villages grâce à l'action de séduction entreprise auprès d'eux par les missionnaires ${ }^{4}$ (Hemming, 1978). Les missions organisent ensuite strictement le travail (rémunéré en principe puisque les Indiens sont libres) et la vie sociale, afin de briser les cultures amérindiennes et de les christianiser.

9 Missions et chasseurs d'esclaves sont donc en concurrence pour la même ressource, et celle-ci diminue rapidement du fait des mauvais traitements mais aussi du fait du choc épidémiologique, qui décime de manière récurrente les ethnies ainsi regroupées. En parallèle, la main-d'œuvre amérindienne est peu prisée dans l'économie des plantations sucrières, et dès le XVI ${ }^{\mathrm{e}}$ siècle des esclaves africains sont importés sur la côte du Brésil, entraînant une marginalisation progressive de l'importance des habitants originels du Brésil pour les Portugais. Au fur et à mesure que leur emprise sur la colonie est assurée et que se développent plantations et mines, le concours des populations locales leur est de moins en moins nécessaire. Le processus prendra toutefois plus de temps en Amazonie, dont l'économie repose sur des collectes forestières qui mobilisent les connaissances indigènes.

10 C'est dans cette région que la compétition autour du travail des Amérindiens sera donc la plus vive au XVIII siècle. En 1755, une loi les émancipe et leur donne des droits équivalents aux citoyens portugais. Sous l'apparente générosité, on trouve l'idée de garantir les avancées diplomatiques obtenues lors du traité de Madrid, qui repose sur l'occupation effective des territoires pour en attribuer la souveraineté. Ainsi, des Indiens « portugais » permettent de conforter la domination lusitanienne sur le Brésil. Mais la question de la mobilisation de la main-d'œuvre est aussi présente, ainsi que le confirme le statut de $1757^{5}$ et les textes du début du XIX ${ }^{\mathrm{e}}$ siècle qui instaurent des brigades de travailleurs obligatoires. La participation des Indiens au soulèvement populaire de la cabanagem, durant la période 1830-1840, est probablement en grande partie née de cette participation forcée aux activités économiques, que l'on retrouvera également durant le boom du caoutchouc. 
11 Le résultat des différents mécanismes de la période coloniale est la modification radicale du peuplement amérindien du Brésil. La population chute vertigineusement, en particulier du fait du choc épidémiologique mais aussi du fait des attaques et des mauvais traitements. De nombreuses ethnies disparaissent totalement, en particulier les importantes civilisations du cours moyen et de l'embouchure de l'Amazone. Les Indiens alliés n'échappent pas aux épidémies, et ils voient aussi leur système culturel et social désarticulé par leurs ravages. Pour beaucoup, ils se fixent autour des noyaux urbains et se métissent peu à peu avec les populations d'autres origines qui s'y trouvent, donnant naissance aux caboclos ou mamelucos qui ont formé une partie importante de la population du Brésil colonial et qui sont demeurés majoritaires en Amazonie jusqu'au $\mathrm{XX}^{\mathrm{e}}$ siècle. Dans certains cas, la disparition n'est qu'apparente, les identités demeurant sous-jacentes mais cessant d'être explicitement revendiquées pour éviter les discriminations qui y sont liées. Les Amérindiens hostiles aux Portugais, eux, sont en général réduits lorsqu'ils tentent de défendre leur territoire. C'est, par exemple, le cas des Muras sur le cours moyen de l'Amazone, décimés et finalement "pacifiés » au XVIII ${ }^{e}$ siècle après cinquante ans de résistance. D'autres ethnies utilisent la fuite et le fait de se réfugier dans des régions toujours plus inaccessibles comme stratégies de survie. On peut citer le cas de la migration des Wajãpi du Xingu jusqu'en Amapá, ou bien celle des Kayapó, venus des savanes du Nordeste et réfugiés dans les forêts du Pará.

\section{De l'Empire à l'Estado Novo : un symbole de brésilianité en voie d'évanescence}

12 L'indépendance du Brésil a peu changé la situation des Amérindiens. L'expansion brésilienne sur leurs terres s'est au contraire accélérée, en particulier à la fin du $\mathrm{XIX}^{\mathrm{e}}$ siècle en Amazonie, avec le boom du caoutchouc, puis au début du $\mathrm{XX}^{\mathrm{e}}$ siècle dans le sud du pays avec l'expansion du front du café ou dans le Nordeste avec celle des plantations de cacao. Leur présence est en général vue comme un obstacle, qu'il s'agit de réduire. Les techniques pour ce faire varient, certaines sont particulièrement vicieuses, comme celle consistant à offrir aux Indiens des vêtements provenant des hôpitaux afin de les contaminer avec la variole ou d'autres maladies connues pour décimer leurs villages (Pereira Gomes, 1988). Dans les années 1930, Pierre Monbeig (1952) évoque encore les « chasseurs d'Indiens » du front du café et Claude Lévi-Strauss montre, au début de Tristes tropiques les effets de la spoliation de leurs terres dans cette même région.

13 Sur le plan idéologique et symbolique cependant, la situation change assez considérablement. Après son émancipation du Portugal, le Brésil cherche en effet à définir une identité propre. Rapidement, l'héritage amérindien est revendiqué comme donnant au pays et à sa population des caractéristiques uniques qui l'ennoblissent. Comme le dit Lilia Schwarcz (2006): «In a slave-based country like nineteenth-century Brazil, only an idealized Indian would do as the symbol of the young nation ». Certains traits prêtés aux Amérindiens comme la force physique, la vaillance, la fidélité ou la connaissance intime de la nature sont associés à cette image et incorporés au récit national. Le fameux roman, puis opéra $O$ Guarani, de José de Alencar, est exemplaire de ce mouvement qui repose sur une production littéraire importante à partir de la 
seconde moitié du XIX siècle - en fort contraste avec le traitement des Amérindiens sur le terrain.

14 Il faut attendre le début du XX $\mathrm{X}^{\mathrm{e}}$ siècle pour que le Brésil commence à mettre en place une politique de protection à leur profit, sous l'impulsion du Maréchal Rondon. Si le Service de protection des Indiens (SPI) a une orientation principalement assimilatrice, il mène une action de recensement des populations concernées (grâce notamment à l'action de l'anthropologue Curt Nimuendaju) qui permet de commencer à systématiser la carte des ethnies au Brésil, en particulier en Amazonie (Hemming, 2003). Ces avancées se concrétisent dans la Constitution de 1934, qui est la première à mentionner les territoires amérindiens comme appartenant légitimement à leurs occupants, tout en interdisant la vente de ces terres. Ces dispositions seront conservées dans celles de 1937 et 1946.

Certains groupes obtiennent donc des territoires qui leurs sont réservés mais à l'exception des Kadiwéu, récompensés pour leur engagement aux côtés du Brésil lors de la guerre du Paraguay, ceux-ci sont en général exigus et ils ne permettent pas le maintien des systèmes traditionnels de subsistance de la plupart des ethnies. Le but de cette politique est de transformer les Amérindiens en travailleurs ruraux pour les intégrer à la matrice brésilienne. Dans les années 1950, sous l'impulsion notamment de Darcy Ribeiro, le SPI prend peu à peu conscience de son aporie, puisqu'en favorisant l'intégration des Amérindiens à la société et à l'économie du Brésil, il détruit l'objet qu'il était chargé de protéger (Devine Guzman, 2013).

16 Le contraste est d'autant plus difficile à accepter que l'incorporation des symboles amérindiens dans la construction de l'identité nationale a été encore renforcée par l'Estado novo, qui décrète par exemple, en 1934, le 19 avril comme " jour de l'Indien " (Garfield, 2000). D'un autre côté les mouvements artistiques continuent d'exalter la participation amérindienne à l'identité brésilienne, par exemple avec le roman Macunaima, de Mario de Andrade.

\section{Des Indiens sauvés par le gong de l'écologie?}

17 La question de l'attribution de territoires protégés pour les Amérindiens commence à se poser sous un jour nouveau à la fin des années 1950. L'idée de laisser de vastes zones aux groupes encore isolés ${ }^{6}$ progresse avec la campagne des frères Vilas Boas pour la création du Parc du Xingu, finalement acceptée en 1961. Bien qu'en retrait par rapport à la demande initiale, ce sont plus de $26000 \mathrm{~km}^{2}$ d'un seul tenant qui sont ainsi officiellement réservés afin de permettre aux habitants de préserver leur mode de vie, l'objectif d'intégration à la société brésilienne étant remis à plus tard (Freire, 1987).

La période de la dictature militaire est marquée une série de mouvements contradictoires sur la question amérindienne. La philosophie du régime revient plutôt à l'assimilation mais convient que celle-ci peut prendre du temps dans le cas des groupes les plus isolés, en particulier en Amazonie. Le principe de territoires réservés est ainsi confirmé par la Constitution de 1967 qui introduit la notion d'usufruit exclusif ${ }^{7}$, et quelques territoires de grande taille sont concédés, comme le «Parc indigène Tumucumaque " à la frontière avec le Surinam. Toutefois, le régime militaire met également en place de grandes infrastructures comme les routes Transamazonienne ou Perimetral Norte qui impactent fortement les ethnies présentes dans les régions concernées. Il recourt parfois au déplacement forcé (cas des Panará du Mato Grosso, 
voir Arnt et al., 1998), voire à des campagnes militaires (contre les Waimiri-Atroari en Amazonas, voir Baines, 1993).

Le statut de l'Indien, promulgué en 1973, est particulièrement représentatif de ces contradictions. De philosophie clairement assimilationniste, considérant les Indiens comme des mineurs sous tutelle, il participe clairement d'un projet assimilationniste. Cela étant, il reconnaît explicitement pour ce faire l'existence de populations amérindiennes non intégrées et crée des droits et une responsabilité pour l'État à leur sujet, dont le mouvement indigéniste saura parfaitement jouer pour forcer la reconnaissance de territoires. En effet, en réaction aux impacts des grands projets d'infrastructure sur les dernières populations amérindiennes isolées, anthropologues, étudiants et une fraction de la société civile se mobilisent sur la "question indigène ». Cette mobilisation, dans laquelle l'Église catholique joue un rôle important, permet la structuration d'ONG d'appui aux droits des Amérindiens et également l'ébauche d'un mouvement amérindien unifié (Belleau, 2014).

Au début des années 1980, ce mouvement et ses alliés associent la question écologique à leur argumentaire (Albert, 1997). Les Indiens sont en effet présentés comme le meilleur rempart contre la déforestation (Conklin et al., 1995), dont le rythme et l'impact commencent à préoccuper sérieusement l'opinion publique mondiale. Cette alliance avec l'écologie permet de démultiplier l'audience des revendications des Amérindiens. Elles ne sont plus cantonnées à un problème social spécifique au Brésil, mais bénéficient aussi d'un soutien mondial (on se souvient du chanteur Sting venant donner sa caution à la lutte des Kayapó contre le barrage de Belo Monte en 1989).

De ce fait, le mouvement pro-Indien se trouve en pleine ascension en 1988, au moment de l'Assemblée constituante. Par une habile mobilisation de certaines ethnies, en particulier les Kayapó, et par un jeu sur le capital symbolique que ceux-ci représentent (Ramos, 1998), il obtient d'importantes avancées, notamment dans la définition des droits fonciers des Amérindiens. Durant les années 1990, les Amérindiens prennent de plus en plus le contrôle de leurs revendications en se dotant d'associations représentatives qui assurent le dialogue avec (ou exercent la pression sur) les organismes gouvernementaux (Albert, 2000; Belleau, 2014). Certaines de ces associations sont puissantes sur le plan local: Conseil indigène de Roraima (CIR), Association Yanomami Hutukara (HAY), Conseil général de la tribu Tikuna (CGTK), etc. ; d'autres sont des coordinations régionales ou nationales: Coordination des organisations indigènes d'Amazonie brésilienne (COIAB), Articulation des peuples indigènes du Brésil (APIB), etc.

\section{L'enjeu des territoires amérindiens}

\section{Fondements légaux}

La Constitution de 1988 offre une véritable rupture par rapport à toutes les politiques précédentes, basées sur le principe d'assimilation, et « fait entrer le pays dans la voie du multiculturalisme » (Boyer, 2010). La nouvelle loi fondamentale affirme en effet le droit des peuples amérindiens à préserver leurs cultures et modes de vie. La question amérindienne y fait l'objet d'un chapitre à part entière (chapitre VIII, « Des Indiens "), et l'article 231 reconnaît aux Indiens non seulement le droit à la préservation de leurs différences culturelles mais aussi des droits sur les territoires qu'ils occupent et sur 
l'ensemble des espaces nécessaires à leur « reproduction physique et culturelle selon leurs us, coutumes et traditions ». Les droits fonciers consistent en un usufruit exclusif, collectif, permanent et incessible des ressources du sol et de l'environnement du territoire en question (le territoire en lui-même demeure propriété de l'État fédéral, qui conserve le droit d'exploitation du sous-sol).

Les territoires amérindiens ne sont donc plus définis comme les terres effectivement occupées à un instant $t$, mais comme l'ensemble des terres nécessaires à la survie physique et culturelle des groupes concernés, incluant dès lors les espaces de réserve pour une occupation future (dans le cas des groupes semi-nomades), les espaces sacrés, les espaces occupés historiquement, etc. C'est sur la base de cette disposition que des territoires de très grande taille leur furent alloués dans les années 1990. La préservation culturelle sous-jacente aux articles de la Constitution sera également la base de droits différenciés dans le domaine de l'éducation (enseignement en langue locale) et de la santé (mise en place d'un système de santé différencié). Ces garanties ont ensuite été complétées par d'autres textes, en particulier par la ratification de la convention 169 de l'Organisation internationale du travail (OIT) (Albert, 2004).

Ces dispositions nouvelles ne sont pas sans créer de débat. Leur ampleur est contestée dès la promulgation de la Constitution. Elle fut restreinte en 1996 avec l'introduction d'une possibilité de contestation des délimitations sur la base de titres de propriété émis antérieurement au processus de reconnaissance ${ }^{8}$. En 2005, tout en confirmant l'homologation de la terre Raposa Serra do Sol, le Tribunal supérieur fédéral dressa par ailleurs une série de conditions appelées, selon lui, à faire jurisprudence: droit des forces armées à se déplacer librement dans les territoires amérindiens, application de la législation environnementale en cas de superposition avec une aire protégée, etc. En 2013, cependant, le même tribunal a reconnu que ces conditions ne valaient que pour le cas analysé et n'avaient pas vocation à redéfinir ou encadrer le droit des territoires amérindiens en général. Pour autant, comme on le verra dans la troisième partie, ce jugement a eu une influence sur d'autres cas en litige auprès des instances judiciaires inférieures (Lages et Teixeira Assis, 2015).

\section{Un patrimoine immense récemment reconnu}

Sous l'égide du ministère de la Justice, la Fondation nationale de l'Indien (FUNAI) est chargée de reconnaître et de délimiter les territoires, qui sont ensuite homologués par le président de la République. Après l'adoption de la Constitution de 1988, on constate un très vaste mouvement de reconnaissances de "terres indigènes " (Le Tourneau, 2006 ; 2014 ; RAISG, 2016) durant les années 1990 et le début des années 2000, comme le montre clairement l'illustration 1. 
Illustration 1 - Homologation de territoires amérindiens par gouvernement depuis 1985

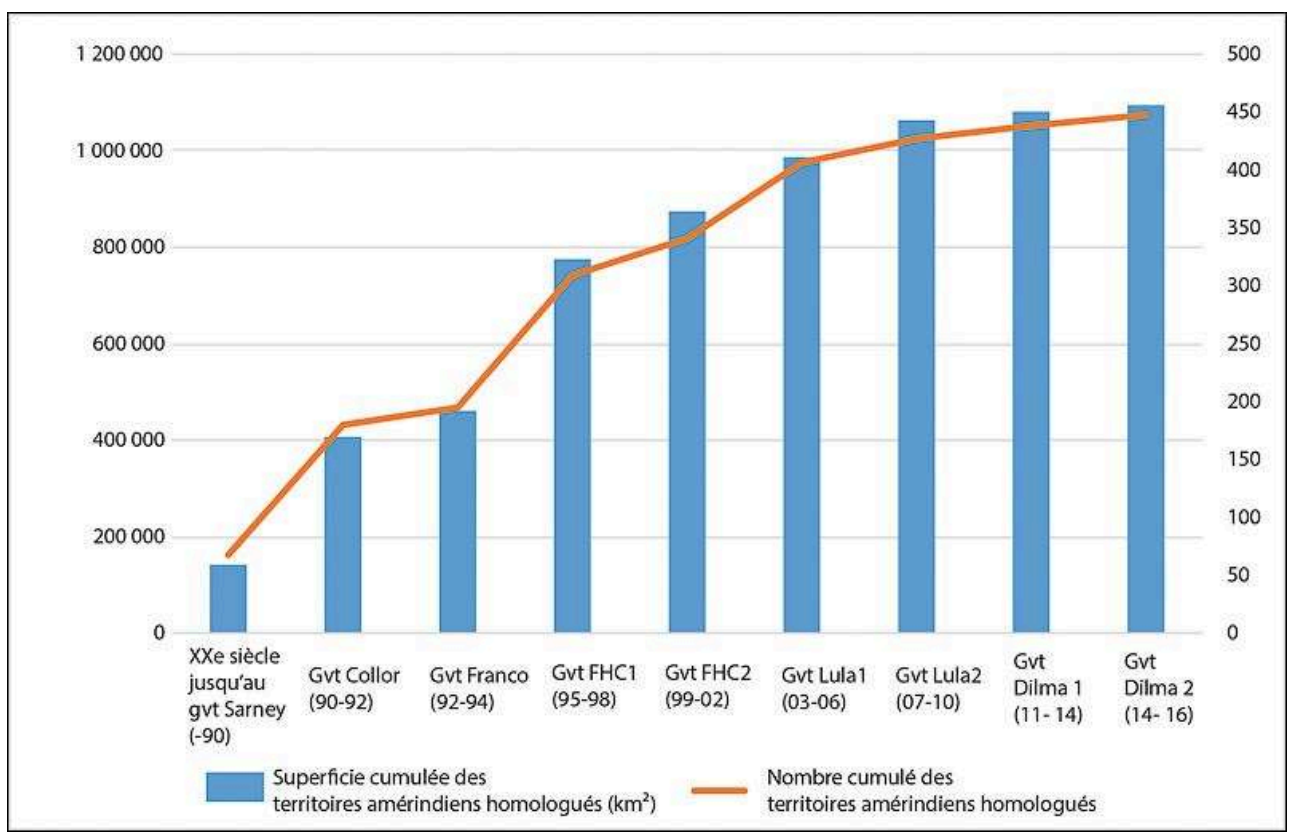

Source : Instituto Socioambiental ; mise en forme : F.-M. Le Tourneau.

Ce augmentation ne résulte pas tellement d'une explosion des revendications, mais surtout d'une consolidation juridique des territoires. Ainsi, en 1990, les territoires en cours d'évaluation par le gouvernement fédéral représentaient un peu moins de $800000 \mathrm{~km}^{2}$ mais seuls $34 \%$ se trouvaient en fin de processus et donc confirmés. En 2015, cette proportion est montée à presque $94 \%$ (pour une extension qui dépasse 1,1 millions de $\mathrm{km}^{2}$ ), ce qui montre l'ampleur des progrès accomplis, notamment grâce à des financements de la communauté internationale (particulièrement de l'Allemagne) via le Programme pilote pour les terres indigènes d'Amazonie légale (PPTAL, voir FUNAI 1999 et 2002). Ces nouvelles reconnaissances se sont concentrées de manière très importante en Amazonie, puisque cette région a absorbé presque toute la différence existant entre 1990 et 2015, passant de 781821 à $1055941 \mathrm{~km}^{2}$, soit 98,4\% de la surface de l'ensemble des territoires amérindiens du Brésil. L'Amazonie est aussi plus avancée sur le plan juridique puisque 78,7\% des territoires (représentant 91,5\% des surfaces) ont atteint le stade de l'homologation contre seulement $52,4 \%$ (63\% des surfaces) dans les autres régions.

La région amazonienne a par ailleurs pour spécificité qu'un certain nombre des terres amérindiennes qui s'y trouvent forment des méga-territoires, soit en eux-mêmes, soit parce qu'ils composent avec leurs voisins des mosaïques dont les superficies sont équivalentes à celles de certains pays d'Europe (illustration 2). On peut ainsi citer la mosaïque des territoires amérindiens de l'Alto Rio Negro ou celle de l'ensemble Xingu/ terres Kayapo qui dépassent chacune $150000 \mathrm{~km}^{2}$. Cela étant, la taille moyenne des territoires amérindiens en Amazonie est bien plus modeste : $2983 \mathrm{~km}^{2}$ (mais contre $91 \mathrm{~km}^{2}$ hors Amazonie). Les causes de la concentration et de l'immensité des territoires amérindiens d'Amazonie sont claires. Par sa difficulté d'accès, l'Amazonie a été la dernière région à être conquise par la société brésilienne. De nombreux groupes amérindiens ont pu y maintenir jusqu'à aujourd'hui leurs systèmes d'usage de l'espace et, lorsque s'est posée la question de la reconnaissance de leurs droits fonciers, il a été 
plus facile de tailler large puisque les terres en question étaient pour leur plus grande partie des terras devolutas, c'est-à-dire des terres publiques sans destination. La question amérindienne au Brésil repose donc sur un contraste très important à l'heure actuelle, entre les Indiens d'Amazonie, souvent plus visiblement éloignés du reste de la société brésilienne et à qui de vastes territoires ont été alloués (parfois justement au motif de cette différence plus visible) et les Indiens du reste du Brésil, dont les « terres indigènes " ont été allouées a minima (le cas des Guarani au Mato Grosso do Sul est particulièrement exemplaire) et ne permettent en aucun cas de maintenir un mode de vie traditionnel.

Illustration 2 - Les territoires amérindiens du Brésil en 2015

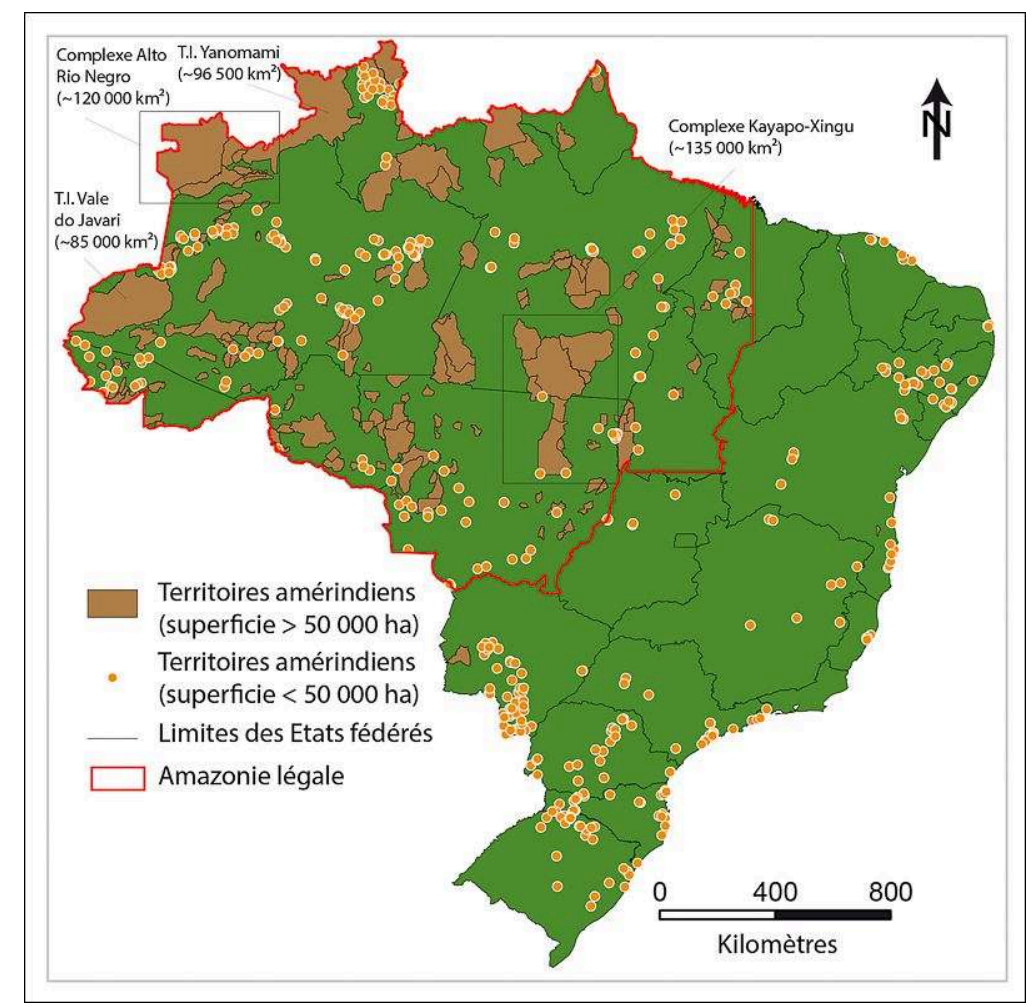

Source : FUNAI ; mise en forme : F.-M. Le Tourneau.

Sur l'ensemble du Brésil, il existe aujourd'hui 596 territoires engagés dans le processus administratif de reconnaissance (dont 480 se situent dans les deux stades finaux). Cet ensemble représente plus de 116 millions d'hectares, ou 13,7\% de la superficie du pays (illustration 2). Il existe par ailleurs un "stock» d'environ 108 autres "en identification » sur lesquels la Fondation Nationale de l'Indien ne s'est pas encore prononcée ${ }^{9}$. Du fait des identités résurgentes, il existe par ailleurs encore un réservoir de demandes potentielles. Le Conseil indigéniste missionnaire (CIMI) parle de près de 150 situations qui n'auraient pas encore été traitées, mais ce chiffre doit être envisagé avec prudence. Quoiqu'il en soit, la question des territoires amérindiens est loin d'avoir été résolue, alors que l'Assemblée constituante avait donné un délai de 5 ans, en 1988, pour terminer le travail... On notera néanmoins que les revendications qui apparaissent désormais concernent le plus souvent des territoires de petite taille et qu'il est probable que le temps de reconnaissances de "terres indigènes" supérieures à un million d'hectares est révolu. Ainsi, le chiffre final de la portion du Brésil réservée aux 
Amérindiens devrait rester durablement autour de 13,7 ou $13,8 \%$ de la superficie du pays.

\section{Une population très minoritaire}

La population amérindienne recensée au Brésil était de 896900 personnes en 2010, soit $0,4 \%$ de la population totale du pays (Le Tourneau, 2014), répartie en 252 ethnies différentes. Ce chiffre montre bien qu'il s'agit d'une minorité très marginale sur le plan démographique. Mais pour faible qu'il soit, il reflète aussi les deux étapes de la démographie amérindienne depuis la conquête coloniale. La première a été une phase de disparition combinant l'élimination physique, le métissage culturel et l'assimilation $^{10}$. Elle s'est étendue jusqu'aux années 1950. La seconde est une phase de récupération intervenue depuis les années 1950. Le recensement de 1991 a ainsi compté 294000 Amérindiens (0,19\% de la population totale), et celui de 2000, 734000 (0,43\%). Ce doublement en proportion, intervenu en moins de dix ans, doit sans doute être attribué principalement au changement de paradigme politique, qui a permis aux Amérindiens de moins hésiter à se reconnaître comme tels, un phénomène appelé "résurgence identitaire ». On peut citer le cas des Tikuna qui se sont longtemps cachés sous l'étiquette de caboclo (Pacheco, 1990).

De 2000 à 2010 la progression en proportion a été marginale, malgré un taux d'accroissement spectaculaire de 4 à $5 \%$ par an chez certaines ethnies comme les Xavante étudié par Coimbra et Welch (2013), ou les Kayabi (Pagliaro, 2010). Cette croissance faible en proportion invite donc surtout à relativiser l'importance numérique du phénomène de résurgence identitaire à l'heure actuelle ${ }^{11}$.

Illustration 3 - Population amérindienne au Brésil par grande région géographique

\begin{tabular}{|l|l|l|}
\hline Région & $\begin{array}{l}\text { Population amérindienne } \\
\text { totale }\end{array}$ & $\begin{array}{l}\text { Proportion de la polulation amériendienne totale } \\
(\%)\end{array}$ \\
\hline Norte & 342836 & 38,2 \\
\hline Nordeste & 232739 & 25,9 \\
\hline Sudeste & 99137 & 11,1 \\
\hline Sul & 78773 & 8,8 \\
\hline $\begin{array}{l}\text { Centro- } \\
\text { Oeste }\end{array}$ & 143432 & 16,0 \\
\hline Total & 896917 & 100 \\
\hline
\end{tabular}

Source : IBGE, Censo Demográfico 2010.

31 Sur le plan régional, le tableau de l'illustration 3 permet de voir que le contingent le plus important se situe dans la région Nord (38,2\%), qui correspond grosso modo à l'Amazonie, et dans le Nordeste (25,9\%). Les autres régions rassemblent à elles trois à peine un peu plus d'un tiers des effectifs. Une certaine logique préside à cette 
répartition : les populations amérindiennes sont plus nombreuses dans les régions qui offraient des zones refuges et qui ont été moins intensivement colonisées que celles du sud (il s'agit aussi des régions les plus vastes).

\section{Le foncier comme moteur du conflit} après la promulgation de la Constitution de 1988 semble s'être peu à peu modifié pour donner lieu aujourd'hui à une conjoncture beaucoup moins favorable.

\section{Le contexte politique : écume des jours ou tendance de fond?}

C'est à partir de l'élection du président Lula et de l'accession au pouvoir du Parti des travailleurs (PT) en 2003 que le climat s'est dégradé. Contrairement aux attentes du mouvement indigéniste, non seulement la ligne idéologique officielle n'a plus fait de la reconnaissance des droits des Amérindiens un axe fort de la politique gouvernementale, mais en plus celle-ci s'est infléchie en faveur d'une politique de construction d'infrastructures et d'équipements (Gomez Bruera, 2013) qui n'était pas sans rappeler celle du gouvernement militaire dans ses objectifs et dans sa philosophie. Le retour de cette sensibilité "développementiste " n'était pas une bonne nouvelle pour les Amérindiens dans la mesure où elle allait se projeter, notamment en Amazonie, sur leurs territoires.

Dès le premier mandat du président Lula on observe un ralentissement du processus de reconnaissance des territoires, amplifié sous la présidence de Dilma Roussef, qui n'aura homologué que 21 «terres indigènes » contre 87 pour le président Lula et 145 pour le président Fernando Henrique (voir illustration 1). En parallèle, un ambitieux projet de réforme du statut de l'Indien est envisagé, le texte de 1973 étant totalement déphasé par rapport à la Constitution de 1988 et à la jurisprudence basée sur celle-ci. Cependant, après la réunion d'une conférence représentative des peuples amérindiens du Brésil en 2007, le gouvernement fédéral a finalement enterré cette initiative pourtant jugée nécessaire par tous. Si la conférence en question a permis la mise en place d'une commission permanente, elle-même remplacée en 2017 par un «Conseil national de politique indigéniste ", censé guider la politique du gouvernement dans ce domaine, l'ouverture est finalement un trompe l'œil puisque la structure du conseil comporte 15 représentants du pouvoir exécutif, qui disposent tous d'un droit de vote, 28 représentants amérindiens dont seulement 13 ont un droit de vote, et 2 représentants d'ONG qui ont un droit de vote. Avec une telle répartition des pouvoirs, les propositions des Amérindiens sont facilement marginalisées.

La question du barrage de Belo Monte a sans doute été le signe le plus visible du divorce entre le PT et les mouvements indigénistes. Alors que le gouvernement a poussé le dossier, intéressé à la fois par le symbole de cette méga-centrale et par ses retombées économiques, les Amérindiens et leurs supporters s'y sont constamment opposés pour des raisons écologiques ou d'ordre culturel (Fearnside, 2017 ; Boanada et al., 2016). Une incompréhension mutuelle s'est manifestée. Du côté du gouvernement et du PT, on mettait en avant «l'absurdité » d'annuler un projet qui allait servir à des millions de Brésiliens "pour seulement quelques dizaines d'Indiens", et le montant des compensations «socio-environnementales » incluses dans le cadre de la construction 
(près de 1,1 milliards d'euros au total). Du côté des Amérindiens, on faisait valoir la valeur inestimable du fonctionnement écologique du Xingu et le patrimoine culturel associé.

Depuis l'impeachment de la présidente Dilma Rousseff et l'arrivée au pouvoir du gouvernement Temer, on peut dire qu'une conjonction forte d'intérêts s'est mise en place en défaveur des Amérindiens, associant au moins trois lobbies actifs au Congrès et dans le gouvernement. Ces groupes de pression sont en particulier le lobby rural, pour lequel les droits fonciers des Amérindiens sont vus comme une menace (en particulier dans le cas du Mato Grosso do Sul, mais aussi d'une manière générale) et qui souhaiterait rendre possible une diminution des territoires alloués pour les convertir en surfaces exploitables. C'est aussi le cas du lobby évangélique, pour des raisons de deux ordres. Sur le plan politico-religieux, d'abord, ce lobby a souvent ciblé les Amérindiens pour des pratiques considérées comme immorales, en particulier les questions d'infanticide. Participer à une redéfinition de la politique indigéniste est donc un moyen de faire avancer leurs conceptions dans ces domaines. Mais d'un autre côté les missions évangéliques sont très fortement engagées dans le système de santé au profit des Amérindiens. Elles sont en contrat avec le gouvernement fédéral pour fournir les services de soin auprès des populations amérindiennes, ce qui permet en même temps de financer et de légitimer leur présence (et leur action de christianisation, même si celle-ci est menée de manière indépendante). Sans vraiment constituer un lobby, la dernière sensibilité politique concernée rassemble les conservateurs de diverses obédiences, en particulier ceux qui sont proches de l'idéologie de la dictature militaire. Pour eux, les territoires amérindiens sont un abandon de souveraineté et induisent un risque de sécession à terme qui ne saurait être toléré. Ils dénoncent souvent l'influence de l'étranger sur ces questions, et y voient une manière d'empêcher le Brésil d'exploiter ses ressources, notamment minières.

Ces trois pôles d'influence ont un poids très important dans la configuration actuelle du Congrès et $\mathrm{au}$ vu des circonstances politiques difficiles, leur appui est absolument nécessaire au gouvernement du président Temer. Malgré le fait que la politique actuelle est particulièrement volatile et réponde autant aux fluctuations à court terme liées aux affaires de corruption qu'à des visions idéologiques, leur influence a été visible dans les dernières nominations à la tête de la FUNAI, qu'il s'agisse de l'éphémère pasteur évangélique Antonio Fernandes Toninho Costa (appuyé par PSC, formation politique évangélique) ou du général de réserve Franklimberg Ribeiro de Freitas (actuellement en poste, soutenu par les conservateurs). Dans ce dernier cas, le gouvernement choisi de nommer un ancien militaire à la tête de la FUNAI, un fait sans précédent depuis la fin de la dictature militaire et un symbole de retour en arrière dont les Amérindiens ne voulaient à aucun prix.

Les changements de personnes sont renforcés par des mouvements de fond. Une nouvelle organisation de l'agence indigéniste a ainsi été proposée dont l'objectif était de renforcer le contrôle du ministre de la Justice sur le travail des groupes techniques chargés d'examiner les demandes de reconnaissance, et même dans une certaine mesure de contester des territoires existants. Il s'agissait clairement d'un geste en direction du lobby rural qui pousse depuis longtemps pour une redéfinition de ces règles. Bien qu'il ait reculé sur certains points de sa réforme, le gouvernement a maintenu l'idée d'un groupe de travail hors de la FUNAI, qui est considérée par beaucoup de parlementaires comme trop favorable aux revendications amérindiennes. 
Enfin, FUNAI et INCRA sont en ce moment l'objet d'une commission d'enquête parlementaire visant à investiguer d'éventuels abus dans l'attribution des terres, dont on devine que l'objectif principal est de contester les homologations des dernières années et paralyser les processus en cours. De fait il existe plus de 20 territoires attendant la sanction finale, dont certains depuis plus de vingt ans, comme la Terre Cachoeira Seca, à proximité de la Transamazonienne.

\section{Jusqu'où les territoires amérindiens sont-ils protégés sur le plan institutionnel?}

Dans ce contexte, on peut se demander quelle est la valeur réelle des droits fonciers des Amérindiens, désormais relativisés ou menacés d'être remis en question par plusieurs mécanismes.

41 Le premier est la conséquence du jugement du Supremo Tribunal Federal (STF) dans le cas de la terre Raposa Serra do Sol, en Roraima. Bien qu'il ait finalement confirmé ce territoire, le tribunal l'a assorti de conditions, qu'il a dans un premier temps souhaité étendre à tous les processus en cours avant de revenir en arrière. Sur cette base toutefois, l'Advocacia geral da União a lancé son décret 303/2012 qui transformait les conditions en question en règle de fonctionnement pour les organes de l'État. Suspendu entre 2012 et 2014 et devant en principe être révisé, ce décret reste pour le moment en vigueur. Il comporte deux principes qui limitent considérablement les possibilités futures des Amérindiens: l'interdiction de révision des limites des territoires déjà homologués et l'introduction d'une "référence temporelle " (Marco temporal). Comme on l'a vu, beaucoup de territoires proposés avant 1988 l'étaient sur la base d'une vision très restreinte des droits fonciers des Indiens, et ils se trouvent pour cela très petits, en particulier dans le sud du pays. En se basant sur la vision plus généreuse de la nouvelle loi fondamentale, certains ont été considérablement augmentés. Ceci ne serait plus possible si le décret est maintenu. Le second point implique désormais de prouver l'occupation et la revendication des zones demandées avant 1988, faute de quoi les demandes sont disqualifiées. Ceci empêche non seulement les revendications liées à la résurgence des identités durant les années 1990-2000, mais complique également la question dans le cas de mouvements de population durant ces mêmes décennies. les Munduruku, illustre bien les difficultés apportées par ce principe (Fearnside, 2015). Il s'agit en effet d'une zone dans laquelle les populations concernées venaient chasser et pêcher depuis des générations, mais où trois villages n'ont été installés que récemment. Comment alors prouver l'antériorité et la légitimité de cette occupation discontinue mais néanmoins ancienne et réelle? Situé dans la zone potentiellement affectée par les barrages envisagés sur le fleuve Tapajos, ce territoire est aujourd'hui contesté, en particulier par l'entreprise Electrobrás, justement sur la base de la "référence temporelle », bien qu'il ait été reconnu par la FUNAI et qu'il ait fait l'objet d'un processus d'auto-démarcation de qualité. De fait, la présence d'un territoire amérindien homologué compliquerait considérablement la construction des centrales envisagées et pourrait même la rendre impossible.

Une autre préoccupation concerne des projets d'amendement à la Constitution. Le projet $\mathrm{PEC} / 215$, qui chemine depuis près de deux décennies au Congrès, vise à donner au Sénat fédéral la haute main sur la reconnaissance des droits fonciers des 
Amérindiens. Selon la plupart des analystes, cette proposition est inconstitutionnelle et elle remettrait en cause la répartition des pouvoirs. La reconnaissance des droits fonciers relève en effet du domaine de l'exécutif (puisqu'elle est une application des droits existants) et non de celui du législatif (puisqu'il ne s'agit pas de la création de droits nouveaux). La proposition semble toutefois regagner de la force actuellement. Une autre proposition d'amendement (PEC 237/2013) vise à autoriser des concessions de terres amérindiennes au profit de fermiers ou d'exploitants forestiers - pratique interdite jusqu'à aujourd'hui en vertu de l'exclusivité de l'usufruit garanti aux Indiens. Même si des cas d'exploitation conjointe existent déjà (par exemple au Mato Grosso où les Parecis cultivent du soja au profit de fermiers locaux), le risque est bien évidemment celui d'une exploitation incontrôlée, notamment du fait du dialogue inégal entre les acteurs du dossier. L'exploitation des diamants chez les Cinta Larga ou celle, passée, de l'or et des bois précieux dans les terres des Kayapó sont là pour rappeler les effets négatifs de telles perspectives.

Qui plus est, une telle inflexion représenterait un changement complet de paradigme par rapport à la politique environnementale du gouvernement fédéral. Avec la promulgation de la "politique de gestion environnementale des territoires amérindiens » en 2007 (Política nacional de gestão ambiental das terras indígenas - PNGATI), le gouvernement avait en effet érigé ces territoires en unités de conservation, permettant au Brésil d'augmenter très substantiellement les surfaces protégées sur son territoire et donc de se présenter de manière favorable dans les négociations sur le climat. Si ces mêmes territoires peuvent être l'objet de concessions et d'une exploitation économique intensive, il faudra donc retirer plus d'un million de $\mathrm{km}^{2}$ des surfaces protégées...

\section{Une situation difficile sur le terrain}

Si l'évolution de la situation sur le plan politique est préoccupante pour les Amérindiens et leurs alliés, la situation sur le terrain l'est plus encore pour plusieurs raisons.

En premier lieu, les invasions dont leurs territoires sont l'objet se multiplient dans un contexte où, minée par le manque de moyens consécutif à la crise économique dont souffre le Brésil, l'action publique peine à faire respecter les lois. Certaines zones sont particulièrement conflictuelles, comme les territoires Awa-Guajá du Maranhão, cible des exploitants forestiers illégaux, ou les rives du fleuve Uraricoera chez les Yanomami, envahies par les chercheurs d'or. Mais on constate également des poussées dans des zones qui étaient jusqu'ici plutôt préservées, comme chez les Uru-Eu-Wau-Wau en Rondônia, où des fraudeurs (grileiros) promeuvent des lotissements illégaux sur leurs terres, ou dans la vallée du Javari où les exploitants forestiers péruviens étendent leurs zones d'activité aux dépends des Indiens Korubo. Parfois les Amérindiens eux-mêmes sont divisés sur la question. L'exploitation du diamant chez les Cinta-Larga de Mato Grosso, et maintenant chez leurs voisins Suruí de Rondônia est ainsi à la fois subie mais aussi acceptée par certains faute d'alternative économique viable. Ces invasions créent aussi un climat de violence contre ceux qui les dénoncent. Selon le CIMI, si 97 leaders amérindiens ont été assassinés en 2007 en lien avec des conflits de terre, ce nombre a augmenté atteignant 138 en $2014^{12}$. 
47 En second lieu, le manque de moyens des administrations rend particulièrement difficile la situation des Amérindiens. Dans le domaine sanitaire, le système consacré à leurs besoins est à bout de souffle. À titre d'exemple, bien que des progrès aient été accomplis, le taux de mortalité infantile était de presque 41,9 pour mille pour les populations amérindiennes en 2010 , contre 15,6 pour la population brésilienne dans son ensemble. La FUNAI elle-même est exsangue. Son budget a été amputé de plus de $50 \%$, que ce soit du fait de coupes ou de contingentements budgétaires, si bien qu'il n'atteint pas 28 millions d'euros pour la gestion de 13,7 \% du territoire brésilien, alors qu'elle doit assurer en plus des missions de délimitation de nouvelles terres et d'assistance au développement des villages... Près de 350 postes ont été supprimés récemment, ce qui a entraîné en particulier la dissolution de plusieurs «fronts de protection ethno-environnementaux" qui avaient été mis en place au profit des groupes les plus isolés. Ceux-ci continuent pourtant d'exister et de chercher à fuir le contact avec la société brésilienne, comme le montre le cas des Kawahiva du nord du Mato Grosso, pour lesquels un territoire a été délimité in extremis grâce à des photographies prouvant leur existence.

\section{Conclusion : beaucoup de terre et peu d'Indiens, le retour d'un vieux slogan}

«Les Indiens, c'est la terre. Et la terre, pour les Indiens, c'est la justice » résume le procureur du ministère public Luciano Mariz Maia ${ }^{13}$, à la tête de la $6^{\mathrm{e}}$ chambre de la Procuradoria geral da República, consacrée aux droits des minorités. Ce faisant, il met bien en valeur la dimension éminemment foncière de la question amérindienne au Brésil. Depuis la colonisation en effet, l'enjeu est bien le territoire, occupé par les Amérindiens selon des modes et une rationalité qui ne sont pas acceptés par le reste de la société brésilienne, et qui fait l'objet de convoitise de la part des fermiers, exploitants forestiers ou entreprises minières. Basée sur une conception de réparation d'un préjudice historique, la Constitution adoptée par le Brésil en 1988 a posé les bases d'une vaste restitution de ces terres, qui fait qu'aujourd'hui près de 13,7 \% de la superficie du Brésil se trouve théoriquement réservé à l'usufruit exclusif des Amérindiens. Le contexte politique actuel a cependant considérablement changé et les droits fonciers qui semblaient acquis sont remis en question.

Plusieurs scénarios sont donc envisageables. Dans le plus favorable, les territoires déjà reconnus se maintiendraient, notamment du fait de la vigilance de la $6^{\mathrm{e}}$ chambre de la Procuradoria geral da República qui a jusqu'ici réussi à maintenir la lettre de la Constitution de 1988 et qui peut contraindre l'exécutif à se mobiliser. En revanche, il faudrait sans doute attendre avant que le retard dans l'examen des reconnaissances de territoires ne soit rattrapé, en particulier pour les plus conflictuels. Un second scénario verrait certains territoires remis en question au moins partiellement, notamment pour assurer la réalisation de certains projets d'infrastructure (par exemple dans la région $\mathrm{du}$ Tapajos, où des barrages sont prévus). Bien que jusqu'ici aucun territoire amérindien n'ait été diminué par le Congrès, celui-ci a déjà agi de la sorte pour des unités de conservation qui disposent pourtant elles aussi d'un niveau de protection élevé. Enfin, le scénario le plus défavorable consisterait en l'approbation de modifications de la Constitution qui modifieraient le régime des territoires amérindiens ouvrant éventuellement la voie à une remise en question beaucoup plus 
générale des terres déjà reconnues. Ce dernier scénario n'est plus improbable aujourd'hui et il montre que les garanties constitutionnelles, autrefois considérées comme absolues, ne valent que si l'on ne modifie pas les règles du jeu.

On pourrait penser que ces considérations juridiques sont secondaires par rapport à une situation sur le terrain dans laquelle orpailleurs, exploitants forestiers ou éleveurs n'hésitent pas à envahir les terres des Amérindiens, voire à s'en prendre directement à ces derniers. Il faut toutefois considérer que la loi est le seul levier disponible pour repousser ces atteintes. En donnant le signal que les droits fonciers amérindiens sont contestables, le pouvoir politique fait entrer d'immenses régions dans une spirale particulièrement inquiétante. Dès lors, la réconciliation qui semblait se profiler après les avancées de 1988 est encore lointaine et le Brésil, s'il continue de faire une place aux Amérindiens dans ses symboles nationaux, est encore très divisé sur la place concrète à leur faire sur son territoire ${ }^{14}$.

\section{BIBLIOGRAPHY}

Albert B., 1997. Territorialité, ethnopolitique et développement : à propos du mouvement indien en Amazonie brésilienne. In Gros C. (ed.), « Amazonies indiennes, Amazonie nouvelles? » Cahiers des Amériques Latines, (23), p. 177-210.

Albert B., 2000. Associações Indígenas e Desenvolvimento Sustentável na Amazônia Brasileira. In Ricardo C. A. (org.), Povos Indígenas no Brasil 1996/2000. São Paulo, Instituto Socioambiental, p. 197-203.

Albert B., 2004. Les Indiens et l'État au Brésil. Problèmes d'Amérique Latine, nº 52, p. 63-84.

Arnt R., Pinto L. F., Pinto R., Martinelli P., 1998. Panará: a volta dos índios gigantes. São Paulo, Instituto Socioambiental.

Baines S., 1993. Censuras e memórias da pacificação Waimiri-Atroari. Brasília, UnB. Série Antropologia, $148 \mathrm{p}$.

Belleau J.P., 2014. Le mouvement indien au Brésil. Rennes, Presses universitaires de Rennes.

Boyer V., 2010. Qu'est-ce que le quilombo devenu ? De la catégorie coloniale au concept anthropologique. Journal de la Société des Américanistes, vol. 96, n² 2, en ligne sur http:// jsa.revues.org/11579.

Boanada V., Leturcq G. et Márcia Grisotti, 2016. Acceptabilité sociale et place de la population lors de la construction du barrage de Belo Monte (Brésil). Éthique publique, vol. 18:1. DOI : 10.4000/ ethiquepublique.2540.

Conklin B. A., Graham L. R., 1995. The Shifting Middle Ground: Amazonian Indians and EcoPolitics. American Anthropologist, 97(4), p. 695-710. Doi : 10.1525/aa.1995.97.4.02a00120.

Coimbra C.E.A., Ventura Santos R., Welch J.R. et al., 2013. The First National Survey of Indigenous People's Health and Nutrition in Brazil: rationale, methodology, and overview of results. BMC Public Health, 13:52. 
Da Matta R., 1976. Quanto custa ser índio no Brasil? Considerações sobre o problema da identidade étnica. Dados, $\mathrm{n}^{\circ} 13$.

Denevan W.M., 2014. Estimating Amazonian Indian numbers in 1492. Journal of Latin American Geography, 13, p. 203-217. Doi :10.1353/lag.2014.0036.

Devine Guzman T., 2013. Native and National in Brazil: Indigeneity after Independence. Chapel Hill, UNC Press.

Fausto C., 2000. Os índios antes do Brasil. São Paulo, Jorge Zahar.

Fearnside P.M., 2015. Brazil's São Luiz do Tapajós dam: The art of cosmetic Environmental Impact Assessments. Water Alternatives, vol. 8(3), p. 373-396.

Fearnside P.M., 2017. Belo Monte: Actors and arguments in the struggle over Brazil's most controversial Amazonian dam. DIE ERDE, vol. 148(1), p. 14-26.

Freire C.A. da Rocha, 1987. Saudades do Brasil ou as lutas pela criação do Parque Indígena do Xingu. Rio de Janeiro , UFRJ/Museu Nacional.

FUNAI, 1999\&2002. Demarcando Terras Indígenas: Experiências e Desafios de um Projeto de Parceria (vol 1 \& 2). Brasília, FUNAI, PPTAL, GTZ.

Garfield S., 2000. As raízes de uma planta que hoje é o Brasil: os índios e o Estado-Nação na era Vargas. Revista brasileira de História, vol. 20, n³9, p. 13-36.

Gómez Bruera H.F., 2013. Lula, the Workers' Party and the Governability Dilemma in Brazil. Routledge, $278 \mathrm{p}$.

Hemming J., 1978. Red Gold: The conquest of the Brazilian Indians. Harvard, Harvard University Press.

Hemming J., 2003. Die If You Must: Brazilian Indians In The Twentieth Century (v. 3). London, Macmillan.

Lages A.S., Teixeira Assis W. F., 2015. A medida do decidir: atuação do STF nos processos de demarcação territorial pós-julgamento da Terra Indígena Raposa Serra do Sol. Novos Cadernos NAEA, v. 18, n 3. Doi: http://dx.doi.org/10.5801/ncn.v18i3.2228.

Le Tourneau F.-M., 2006. Enjeux et conflits autour des territoires amérindiens d'Amazonie brésilienne. Problèmes d'Amérique latine, n 60, p. 71-94.

Le Tourneau F.-M., 2014. En marge ou à la marge : les populations amérindiennes dans le Brésil contemporain. Espaces, Populations et Sociétés, 2014/2-3 | 2015, URL : http://eps.revues.org/5859

Le Tourneau F.-M., 2016. Quelle durabilité pour les territoires amérindiens d'Amazonie brésilienne ? Journal de la société des américanistes, 102-1 | 2016, 167-193. URL : http:// jsa.revues.org/14673

Monbeig P., 1952. Pionniers et planteurs de São Paulo. Paris, A. Colin.

Pacheco de Oliveira J., 1990. Les Tikuna et leur lutte pour la terre. Ethnies, n 11-12, p. 136-142.

Pagliaro H, 2010. The demographic revolution among Brazilian Indigenous peoples: the case of the Kayabi in the Xingu Indian Reservation, Mato Grosso State, Brazil, 1970-2007. Cadernos De Saude Publica, 26(3), p. 579-590.

Pereira Gomes M., 1988. Os índios e o Brasil. Editora Vozes.

Perone Moises B., 1992. Índios livres e índios escravos: os princípios da legislação indigenista do período colonial (séculos XVI a XVIII). In Cunha M. Carneiro da (org.), História dos índios no Brasil, São Paulo:, Companhia das Letras, Secretaria Municipal de Cultura, FAPESP, p. 115-132. 
Pinto de Medeiros R., 2011. Concessão de títulos nobiliárquicos às lideranças indígenas na luta contra invasores estrangeiros e formação de uma elite militar nas capitanias do norte da América Portuguesa (sécs.XVII e XVIII). Anais do XXVI Simpósio Nacional de História - ANPUH, São Paulo, julho 2011.

RAISG, 2016. Cartografía Histórica de Áreas Naturales Protegidas y Territorios Indígenas en la Amazonía. Ramos A., 1998, Indigenism Ethnic Politics in Brazil. Madison, University of Wisconsin Press, 336 p. Ribeiro D., 1996[1970]. Os Índios e a civilização: a integração das populações indígenas no Brasil moderno. São Paulo, Companhia das letras.

Schwarcz L.M., 2006. A Mestizo and Tropical Country: The Creation of the Official Image of Independent Brazil. Revista Europea de Estudios Latinoamericanos y del Caribe, 80, | 25.

\section{NOTES}

1. Soit 1,16 millions de $\mathrm{km}^{2}$, chiffre FUNAI 2017 incluant les territoires ayant parcouru au moins une étape du processus de reconnaissance officielle.

2. Bien que l'estimation de la population amérindienne présente au début du XVIe siècle, la tendance actuelle est de considérer que les chiffres divulgués jusqu'aux années 1980 ont été minorés. Dans un article faisant le point sur la question Denevan (2014) propose jusqu'à 10 millions pour les terres basses de l'Amérique du sud.

3. Produits de la forêt.

4. L'attraction de ces villages, en général situés dans le cours supérieur des rivières, était appelée " descente».

5. Désigné sous le nom de « directoire » car les Amérindiens sont désormais tenus de résider dans des villages dirigés par des «directeurs» civils qui attribuent leur travail en fonction des demandes des colons ou de l'administration.

6. Au lieu de territoires de petite taille limités aux villages et aux terres qui les entourent immédiatement.

7. Cette notion permet de dissocier l'usage des terres et leur propriété, résolvant la délicate question de la vente éventuelle de leurs terres par les Amérindiens. Ceux-ci disposent donc de l'exclusivité de l'usage de l'espace mais pas de la propriété qui demeure l'apanage du gouvernement fédéral - une figure plus acceptable également pour les militaires soucieux de ne pas créer à terme des embryons de territoires indépendants au sein du Brésil.

8. Décret 1.775 de 1996, voir Albert, 1997.

9. Chiffres de l'Instituto socioambiental, mise à jour de juin 2017.

10. Certaines études génétiques (à prendre toutefois avec précautions) vont jusqu'à dire qu'un tiers des Brésiliens possède un patrimoine génétique qui indique des ancêtres amérindiens.

11. Cela étant le stock de revendications reste important puisque plus de $40 \%$ des Indiens vivent en dehors de territoires reconnus.

12. Voir le rapport de la rapporteure spéciale des Nations Unies Victoria Tauli-Corpus après sa mission d'information en 2016 : https://nacoesunidas.org/wp-content/uploads/2016/03/SR-onIPs-end-of-mission-statement-Brazil-17-03-2016-final.pdf

13. "Índio é terra. E terra, para o índio, é Justiça". Entretien avec le journal Carta Capital, 19/04/2107, voir https://www.cartacapital.com.br/sociedade/o-atual-governo-esta-decidido-a-destruir-afunai

14. Symbole bien brésilien de ce débat, les paroles de la samba de l'école Imperatriz Leopoldinense pour le carnaval de 2017, consacré à la région du Xingu, ont fait l'objet d'une 
polémique avec le lobby rural car elles évoquaient les « destructions » réalisées par les fermiers au détriment des Indiens.

\section{ABSTRACTS}

The 1988 Constitution of Brazil, which recognizes extensively indigenous land rights, seemed to make possible the reparation of the historical prejudice represented by the territorial dispossession of Indigenous peoples. However, after 30 years, the reconciliation did not take place. Vast swaths of Brazil (about 12.3\%) were recognized as Indigenous territories, but land conflicts are still acute. The political and social contexts have changed much in the last 10 years, leading to a renewed contestation of claimed but also already established indigenous lands. This paper analyzes the current situation and what is at stake with the many legislative projects under review. To that end, we first sketch a rapid history of how indigenous rights were considered throughout Brazilian history. We then show how the advances linked with the 1988 Constitution have permitted the constitution of a vast amount of "indigenous lands", even if the indigenous population of Brazil only amounts to a very small fraction of Brazilian population. Last, we turn to the current situation and show how indigenous land rights have been more and more contested from 2005 on and how their juridical and physical protection is deeply challenged. In conclusion, we try to trace what future evolution on this topic might be.

En ouvrant de larges droits fonciers aux Amérindiens, la Constitution de 1988 avait semblé avancer dans le sens de la réparation du préjudice historique représenté par la dépossession territoriale des Amérindiens. Trente ans plus tard, force est de constater que la réconciliation attendue n'a pas eu lieu. Le moteur principal de la dispute est sans aucun doute d'ordre spatial. Les Amérindiens disposent en théorie aujourd'hui d'un usufruit exclusif sur une portion considérable du territoire brésilien (environ 13,7 \%) mais le balancier semble avoir effectué son mouvement en sens inverse depuis une dizaine d'années. Pour faire le point sur cette question et sur les enjeux qui lui sont liés, cet article propose tout d'abord un rapide panorama historique de la manière dont la question amérindienne a été considérée au Brésil depuis la colonisation portugaise jusqu'aux avancées de la fin $\mathrm{du} \mathrm{XX}^{\mathrm{e}}$ siècle, en nous focalisant principalement sur la question des territoires. Dans un deuxième temps, nous montrerons comment la Constitution de 1988 a permis la consolidation d'un patrimoine foncier très important, alors que la population amérindienne demeure ultra-minoritaire. La dernière partie fait le point sur la situation actuelle en essayant de montrer comment l'inflexion dans un sens défavorable aux droits fonciers des Amérindiens est patente depuis plus de dix ans et combien la situation actuelle met à l'épreuve les protections dont ils bénéficient. En conclusion, on s'interroge sur les scénarios possibles pour les prochaines années.

\section{INDEX}

Keywords: Brazil, Indigenous people, Indigenous land, land rights, history

Mots-clés: Amérindien, droit foncier, Brésil, territoire amérindien 


\section{AUTHOR}

\section{FRANÇOIS-MICHEL LE TOURNEAU}

François-Michel Le Tourneau, fmlt@fmlt.net, est Directeur de recherches à l'UMI 3157 - iGLOBES , CNRS-University of Arizona, Tucson, USA ; Il a publié récemment :

- Le Tourneau F.-M., 2016. Quelle durabilité pour les territoires amérindiens d'Amazonie brésilienne ? Journal de la société des américanistes, 102-1 | 2016, 167-193. http://jsa.revues.org/ 14673

- Brondizio E., Le Tourneau F.-M., 2016. Environmental governance for all. Science, American Association for the Advancement of Science, 352 (6291), p. 1272-1273.

- Le Tourneau F.-M., 2014. En marge ou à la marge : les populations amérindiennes dans le Brésil contemporain. Espaces, Populations et Sociétés, 2014/2-3 | 2015. http://eps.revues.org/5859 